\title{
Wenyang-Huayin, a Chinese Medicine formulation, inhibits inflammation in OVA-Induced asthma rats
}

\section{Peizheng Yan}

Shandong University of Traditional Chinese Medicine

Qingxiang Zhang (D 1323113659@qq.com)

Shandong University of Traditional Chinese Medicine https://orcid.org/0000-0003-1171-8950

\section{Zhu Qingjun}

Shandong University of Traditional Chinese Medicine

\section{Xing Chang}

Shandong University of Traditional Chinese Medicine

\section{Meng Qingyan}

Shandong University of Traditional Chinese Medicine

Yan Liu

Shandong University of Traditional Chinese Medicine

\section{Research}

Keywords: anti-airway inflammation, asthma, pulmonary, traditional Chinese medicine, Wenyang-Huayin formulation

Posted Date: January 5th, 2022

DOI: https://doi.org/10.21203/rs.3.rs-1207327/v1

License: (9) (1) This work is licensed under a Creative Commons Attribution 4.0 International License. Read Full License 


\section{Abstract}

Background and Objectives

Asthma is a severe public health issue since it causes a progressive decline of pulmonary function and a lot of unnecessary deaths. Traditional Chinese medicine has played a great role in the prevention and treatment of asthma, Wenyang-Huayin (WYHY) formulation is one of the useful prescriptions in clinical, which the pharmacological mechanism has not yet been revealed. In this study, we explored its anti-airway inflammation and pulmonary protection effects in the treatment of asthma induced by ovalbumin (OVA), to provide support for its deeply clinical applications.

Methods

Asthma model with the syndrome of accumulation of cold fluid in the lung in chronic bronchitis (a common type of Traditional Chinese Medicine (TCM) syndrome) was induced in SPF male Wistar rats by sensitizing and challenging with OVA, followed by drink, body cold and fatigue stimulation. WYHY was orally administered at $9.82142857 \mathrm{~g} \cdot(\mathrm{kg} \cdot \mathrm{d})^{-1}$ body weight for 21 days, Rapamycin and the other two traditional Chinese medicines (Xiaoqinglong decoction and Astragalus granules) were used as positive controls. After intervention, the pulmonary function was measured by AniRes 2005 Animal pulmonary function Analysis system, the pathological features of pulmonary alveolus and bronchus were observed after Hematoxylin-Eosin (H\&E) staining, and serum interleukin-13 (IL-13), transforming growth factor - $\beta 1$ (TGF- $\beta 1$ ), tumor necrosis factor- $\alpha$ (TNF- $\alpha$ ), and interferon- $\gamma$ (IFN- $\gamma$ ) levels were determined by enzyme linked immunosorbent assay (ELISA) assay.

Results

The model rats showed heavy signs of airway inflammation and remodeling like chronic asthma, evidenced by the damaged pulmonary alveolus structure, increased inflammatory cell infiltration, declined pulmonary function, and abnormal changes of cytokines. All treatment methods could slow down the damage caused by molding factors, and WYHY might have an advantage in pulmonary protection effects than the others.

Conclude

WYHY has a good effect on anti-airway inflammation and protecting pulmonary function in the treatment of OVA-Induced asthma rats, and its mechanism is worth of in-depth study.

\section{Introduction}

Asthma has become a severe public health issue in various countries over the world ${ }^{1}$. As a health problem for all ages and with approximately 300 million patients worldwide, asthma leads to 250,000 annual deaths, most of which, however, are preventable 2,3. Bronchial asthma is an allergic reaction disease induced by a variety of factors and is a chronic airway inflammatory response involved in multiple cells and cell components, usually associated with Th2 cell mediated eosinophilic airway inflammation. Airway hyperresponsiveness and chronic airway inflammation are the main characteristics of asthma. Asthma is closely related to environmental factors, allergies, airway inflammation, airway hyperresponsiveness, nerve, and other factors ${ }^{4}$. However, chronic inflammation can make the aggravation of airway responsiveness, lead to a variety of reversible airflow limitations, or cause recurrent symptoms such as puffing, chest tightness, or coughing and phlegm. Respiratory tract inflammation is the pathological basis of asthma. Therefore, the control of airway inflammation is the focus of asthma treatment in both acute and chronic remission stages of asthma.

The imbalance between Th1 and Th2 cells' immune responses is a main property of inflammatory asthma. The typical Th1 cell-derived cytokines include IFN- $\gamma$, IL-1, IL-2, and TNF- $\beta$, and the typical Th2 cell-derived cytokines are IL-4, IL-5, TNF- $\alpha$ and IL-13 5 . Previous studies have demonstrated that Th2 cells are vital in the pathogenesis of asthma, and Th2 cell-derived cytokines are closely related to mucus hypersecretion, eosinophil (EOS) accumulation, hyperresponsiveness (AHR) development, and lung remodeling 6,7.

Additionally, TGF- $\beta 1$, mainly produced by EOS ${ }^{8}$, plays a pivotal role in secreting and mediating a mass of growth factors, and cytokines cause airway inflammation and airway remodeling ${ }^{9}$. Expression of TGF- $\beta 1$ is correlated with basement membrane thickness, fibroblast number, and inflammation severity ${ }^{10}$.

Page 2/15 
Despite inhaled corticosteroids, asthma patients still show a decrease in the level of pulmonary function ${ }^{11}$. In recent decades, the development of traditional Chinese medicine has made great progress. It has played a great role in the prevention and treatment of asthma, and the clinical symptoms have been clearly improved ${ }^{12-14}$.

Wenyang-Huayin (WYHY) formulation is a useful prescription for asthma in clinical. In this study, we explored the effects and properties of WYHY in the treatment of asthma induced by OVA and aim to provide support for its deeply clinical applications.

\section{Materials And Methods}

\subsection{Animals.}

SPF male Wistar rats (Weight:200 $\pm 20 \mathrm{~g}$ ) were provided by Jinan Pengyue Experimental Animal Breed Co., Ltd. (permit number: SCXK(Lu)2014-0007). The rats were kept in the laboratory Animal Center of Shandong University of Traditional Chinese Medicine (Jinan, China). Rats were kept in standard living conditions [room temperature $(21 \pm 2){ }^{\circ} \mathrm{C}$, regular humidity $(50 \pm 10 \%)$, and $12 \mathrm{~h}$ darklight cycle]. All experiments were performed in compliance with the guidelines for the care and use of laboratory animals as approved by the Animal Ethics Committee of Shandong University of Traditional Chinese Medicine.

\subsection{Drugs.}

WYHY was prepared from Ephedra, Cassia Twig, Radix Paeoniae Alba, Rhizoma Zingiberis, Schisandra Chinensis, Rhizoma Pinellinae Praeparata, Liquorice, Asarum sieboldi Mig, Ginseng, Astragalus mongholicus, these herbs were mixed in the proportion of 2:2:2:2:2:2:2:1:2:5. Xiaoqinglong Decoction was prepared from Ephedra, Cassia Twig, Radix Paeoniae Alba, Rhizoma Zingiberis, Schisandra Chinensis, Rhizoma Pinellinae Praeparata, Liquorice, Asarum sieboldi Mig, these herbs were mixed in the proportion of 2:2:2:2:2:2:2:1 and extracted by boiling water twice ( $2 \mathrm{~h}$ each time). The decoction was concentrated and dehydrated in vacuo $\left(70^{\circ} \mathrm{C}\right)$ and ground into powder. The extraction rate of the dry extract was $20.29 \%$ (actual dry powder/actual crude herbs). The prepared powder was kept at $0^{\circ} \mathrm{C}-4^{\circ} \mathrm{C}$ and dissolved with purified water into a suspension of different concentrations. Astragalus granules (crude drug proportion 1:10). These herbs were from Huarun three-nine Medicine Co.,Ltd., in Jinan. Rapamycin (100 mg/bottle) were obtained from MedChem Express in America (\#HY-10219)

\subsection{Reagents.}

The reagents used in this study include the following: Aluminum hydroxide, Sigma Inc., St Louis, United States (\#141009001); Ovalbumin, Solarbio, Beijing, China (\#A8040); the following products were from ExCell Bio-Technology Co., Ltd., Taichang, China: rat IL13 ELISA kit (\#ERC15), rat TGF-ß1 ELISA kit (\#ER013-96), rat TNF-a ELISA kit (\#ER006), rat IFN-y ELISA kit (\#ER005-96).

\subsection{Main instruments}

The main instruments were as follows: AniRes2005 Animal pulmonary function Analysis system (Bestlab High-Tech Co., Ltd. Beijing, China); W001 Altrasonic atomizer apparatus (Fulin Medical Equipment Co., Ltd. Jiangsu, China); Leica RM2265 microtome (Leica, Germany); CX23 optical microscope (OLYMPUS, Japan); Ultrapure water preparation system, Milli-Q (Millipore, United State); CT15RT low-temperature high-speed centrifuge (Techcomp, United States).

\subsection{Induction of the syndrome by accumulation of cold fluid in the lung in chronic bronchitis asthma rat model}

Forty-eight male rats were randomly divided into six groups (8 rats per group): a normal group (group 1), a model control group (group 2), a rapamycin group (group 3), Xiaoqinglong Decoction group (group 4), WYHY group (group 5), Astragalus granules group (group 6). Establishment of asthma models were performed according to previous reports ${ }^{[31-33]}$. Briefly, rats of groups $2,3,4,5$, and 6 were injected intraperitoneally (i.p.) with a suspension containing $100 \mathrm{mg}$ OVA and $100 \mathrm{mg} \mathrm{Al}(\mathrm{OH})_{3}$ dissolved in $1 \mathrm{~mL}$ saline on days 1 and 8. Meanwhile, the rats in the normal saline group received $0.5 \mathrm{~mL}$ saline. Every two days during days 15 to 22 , rats of groups other than the normal saline one were stimulated by $1 \%$ atomized OVA for $30 \mathrm{~min}$; saline was used instead of OVA for rats of the normal saline group. Drink cold body cold, fatigue stimulation were performed for 20 days, except the first and 8th day, 30 min before OVA exposure. Rats were sacrificed at day 23.

Induction of the syndrome of accumulation of cold fluid in the lung in a rat model: (1) Drink cold stimulation is to feed rats with ice water mixture daily; (2) Body cold stimulation is to freeze rats in $-10{ }^{\circ} \mathrm{C}$ refrigerator for 3 hours daily; (3) Fatigue stimulation is that rats swim in water at $24^{\circ} \mathrm{C}$, until the nose will sink into the water unable to float, then the rats get out. (Figure 1A)

Page 3/15 


\subsection{Drug intervention}

According to the drug dosage conversion formula between rats and human, rats in the norm and model control group were given purified water and rats in the rapamycin group were given rapamycin suspension $0.013 \mathrm{mg} \cdot(\mathrm{kg} \cdot \mathrm{d})^{-1}$. The rats in the groups of Xiaoqinglong decoction, WYHY were treated with powder suspension of modified Xiaoqinglong decoction, WYHY equal to the crude drug of $6.69642855 \mathrm{~g} \cdot(\mathrm{kg} \cdot \mathrm{d})^{-1}$ and $9.82142857 \mathrm{~g} \cdot(\mathrm{kg} \cdot \mathrm{d})^{-1}$. the rats in Astragalus granules group were given Astragalus granules $0.223214286 \mathrm{~g} \cdot(\mathrm{kg} \cdot \mathrm{d})^{-1}$. Rats were weighed once a week for adjusting drug dosage in the 3 weeks' the intervention period.

\subsection{Sample collection and test}

At the end of the three-week treatment period, rats were anesthetized with pentobarbital sodium solution (4\%, intraperitoneal injection) after $12 \mathrm{~h}$ fasting, then the pulmonary function was measured by AniRes 2005 Animal pulmonary function Analysis system Inspiratory resistance (IR), expiratory resistance (ER) and pulmonary ventilation adaptability (Cldyn). And blood samples were collected from the abdominal aorta, and the left lung tissue was quickly removed, cleaned with saline rinse, and frozen immediately in $-80^{\circ} \mathrm{C}$ refrigerator for cryopreservation.

\subsection{Enzyme-linked immunosorbent assay (ELISA)}

The blood serum samples from the abdominal aorta were centrifuged to collect serum. IL-13, TGF- $\beta 1$, TNF-aand IFN- $\gamma$ were measured by an ELISA assay.

\subsection{Hematoxylin-Eosin staining}

Isolated lungs were fixed with $4 \%$ paraformaldehyde and embedded in paraffin. Then we cut the specimens into a thickness of 3-4 $\mu \mathrm{m}$ sections. The sections were stained with hematoxylin and eosin (H\&E). The results were visualized using a microscope. Three pathologists individually assessed all staining results.

\subsection{Western blot detection}

The expressions of LC-3B, beclin-1, p-mTOR, and mTOR were detected by Western blot. RIPA lysate was used to extract total protein. The samples were taken for sodium dodecyl sulfate (SDS) - polyacrylamide gel electrophoresis in a buffer system. Then the protein in the gel was transferred to the nitrocellulose membrane. The membrane was sealed with $5 \%$ skimmed milk at room temperature for 90 min. Then, the membrane was washed with TBST buffer for 3 times and incubated with the first antibody overnight at $4^{\circ} \mathrm{C}$. After washing with TBST buffer, the corresponding secondary antibody was added the next day and incubated at room temperature for $2 \mathrm{~h}$. At last, the protein bands were visualized using Clarity ${ }^{\mathrm{TM}}$ Western ECL Substrate (Bio-Rad) and then analyzed using ImageJ v1.40 software (Hercules, CA, USA).

\subsection{Transmission electron microscope (TEM)}

The autophagosomes in lung sections were observed by TEM. In brief, the pathological sections of the lung in each group were fixed with $2 \%$ glutaraldehyde buffered with $0.2 \mathrm{mmol} / \mathrm{L}$ cacodylate and postfixed in osmium tetroxide before embedding in epoxy resin for electron microscopy.

\subsection{Statistical analysis}

All data were expressed as mean \pm SD and analyzed with SPSS13.0 software. Differences of parametric data among several groups were determined by one-way analysis of variance (ANOVA). Any differences with $<0.05$ were considered significant (two-tailed).

\section{Results}

\subsection{Effect of WYHY on general conditions}

Rats in Group 1 had the following characteristics: body strong, normal daily food and water intake, normal excretion of urine and stool, smooth fur in good mental state, and swift action, respiration rhythm heat. Rats in Group 2 had the following characteristics: body emaciation, rough and lusterless fur, or thinning limbs, not warm, nose, ears and tail, pale, enuresis, loose stools, ecphysesis, pants, wheezing, white secretion on mouth and nose, mental fatigue, slow in reacting or agitated state, and weight loss (Figure 1B). Compared with those in Group 2, rats in the three herbal medicine decoction groups (groups 4, 5, and 6) showed significantly relieved 
symptoms of ecphysesis, pants, wheezing, and weight loss (Figure 1B), with glossy fur and swift response. The rats in Group 3 also showed improvement of symptoms and signs, although not as significant as those in the three herbal medicine decoction groups.

\subsection{WYHY improved pulmonary function}

As shown in Table 1, the baselines of inspiratory resistance (Ri), expiratory resistance (Re), and lung compliance (Cldyn) were similar between groups $(P>0.05)$. On the 23rd day, Ri and Re in group 2 were significantly higher than group 1 , while Cldyn in group 2 was significantly lower than group $1(P<0.01)$; Ri and Re in the treatment groups (including groups $3,4,5,6)$ were significantly lower than Group 2, while Cldyn in the treatment groups was significantly higher than group $2(P<0.01)$. In addition, there was no significant difference in Ri and Re between the treatment groups $(P>0.05)$, while Cldyn in group 5 was higher than group $3(P<0.05)$ and group 6 $(P<0.01)$.

Table 1

Rats in different groups with pulmonary function on before model

\begin{tabular}{|c|c|c|c|c|c|c|c|}
\hline \multirow[t]{2}{*}{ Groups } & \multirow[t]{2}{*}{$\mathbf{n}$} & \multicolumn{2}{|c|}{$\mathrm{Ri}\left(\mathrm{cm} \mathrm{H}_{2} \mathrm{O} \cdot \mathrm{s} / \mathrm{mL}\right)$} & \multicolumn{2}{|c|}{$\operatorname{Re}\left(\mathrm{cm} \mathrm{H}_{2} \mathrm{O} \cdot \mathrm{s} / \mathrm{mL}\right)$} & \multicolumn{2}{|c|}{ Cldyn $\left(\mathrm{mL} / \mathrm{cm} \mathrm{H} \mathrm{H}_{2} \mathrm{O}\right)$} \\
\hline & & Baseline & Day 23 & Baseline & Day 23 & Baseline & Day 23 \\
\hline $\begin{array}{l}\text { Group } \\
1\end{array}$ & 8 & $1.965 \pm 0.314$ & $1.999 \pm 0.206$ & $1.866 \pm 0.315$ & $1.871 \pm 0.233$ & $0.209 \pm 0.028$ & $0.206 \pm 0.025$ \\
\hline $\begin{array}{l}\text { Group } \\
2\end{array}$ & 8 & $1.985 \pm 0.252$ & $3.502 \pm 0.533^{* \star @ @ ~}$ & $1.846 \pm 0.331$ & $3.589 \pm 0.575^{\star \star @ @ ~}$ & $0.204 \pm 0.016$ & $0.056 \pm 0.016 * @ @$ \\
\hline $\begin{array}{l}\text { Group } \\
3\end{array}$ & 8 & $1.979 \pm 0.345$ & 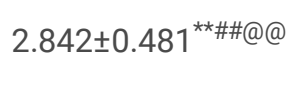 & $1.868 \pm 0.234$ & $2.744 \pm 0.503^{\star \star \# \# @ @ ~}$ & $0.204 \pm 0.022$ & 0.137士0.029*\#\#@@ \\
\hline $\begin{array}{l}\text { Group } \\
4\end{array}$ & 8 & $1.962 \pm 0.262$ & $2.656 \pm 0.407^{\star \star \# \# @ @ ~}$ & $1.896 \pm 0.275$ & $2.486 \pm 0.368^{\star \star \# \# @ ~}$ & $0.204 \pm 0.024$ & $0.166 \pm 0.040^{* \# \# @ ~}$ \\
\hline $\begin{array}{l}\text { Group } \\
5\end{array}$ & 8 & $1.985 \pm 0.240$ & 2.361士0.542\#\#@@ & $1.876 \pm 0.338$ & $2.430 \pm 0.484^{\star \# \# @ ~}$ & $0.207 \pm 0.016$ & $0.195 \pm 0.043^{\# \# \Delta}$ \\
\hline $\begin{array}{l}\text { Group } \\
6\end{array}$ & 8 & $1.963 \pm 0.366$ & $2.779 \pm 0.426^{\star \star \# \# @ @ ~}$ & $1.888 \pm 0.216$ & $2.655 \pm 0.483^{\star \star \# \# @ @ ~}$ & $0.209 \pm 0.022$ & 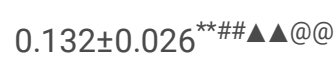 \\
\hline
\end{tabular}

Data were expressed as mean $\pm S D, n=8$.

Group 1: normal group; group 2: model control group; group 3: rapamycin group; group 4: Xiaoqinglong Decoction group; group 5: WYHY group; group 6: Astragalus granules group.

Compared with group $1,{ }^{\star} P \otimes 0.05,{ }^{\star *} P \otimes 0.01$;Compared with group $2,{ }^{\#} P \otimes 0.05,{ }^{\#} P \otimes 0.01$; Compared with group $3,{ }^{\Delta} P \otimes 0.05$; Compared with group 5, $\triangle \triangle P \otimes 0.01$; Compared with Baseline, @P区0.05, @@P凶0.01.

\subsection{WYHY alleviated the pathological injury of lung}

The histopathological features of each group were shown in Figure 2. In group 1, the pulmonary alveolus structure was intact, with rare inflammatory cell infiltration around. The bronchial smooth muscle was normal, with lumen patency and cilia regularly arranged. In group 2, there was a lot of inflammatory cell infiltration around the bronchus and epithelial cells of the bronchial mucous membrane, especially lymphocytes and EOS, and there were also inflammatory cells and increased mucus content inside the bronchial lumen. The alveolar walls are thickened with blood cells and pulmonary alveolus seeped. In group 3 and group 6, although the general pathology feature of pulmonary alveolus and bronchus was better than that in group 2, but there were still a lot of inflammatory cell infiltrations around the bronchus, epithelial cells of bronchial mucous, and inside the bronchial lumen. In group 4 and group 5, the epithelial cells of the bronchial mucous membrane were slightly swelled, and the bronchial smooth muscles were slightly thickened, and there were little inflammatory cells and mucus content seeped around the bronchus and inside the bronchial lumen. In addition, group 5 got the least inflammatory cell infiltration and luminal stenosis. These results suggested that all treatment methods might slow down the damage caused by molding factors, and WYHY group was the best in both groups.

\subsection{WYHY reduced the levels of circle cytokines}


Compared with group 1, the levels of IL-13, TGF- $\beta$, and TNF-a in group 2 increased, while the level of INF- $\gamma$ decreased $(P<0.05$ or $P<0.01)$. Compared with group 2 , the IL-13, TGF- $\beta$, and TNF- $\alpha$ in the treatment groups were all decreased, while the level of INF- $\gamma$ increased $(P<0.05$ or $P<0.01)$. There was no significant difference in IL-13, TGF- $\beta$, TNF-a, and INF- $\gamma$ levels between the treatment groups ( $P>0.05)$ (Figure 3).

\subsection{WYHY activated cell autophagy in lung}

We noticed a decrease in the number of autophagosomes in the lung tissue of asthma rats, and all treatment groups might affect autophagy (Figure 4). Therefore, we determined the autophagy associated proteins LC-3II, LC-3I, beclin 1, and p-mTOR (Figure 5). Compared with group 1, the expression levels of LC-3II/ LC-3I and beclin 1 in group 2 increased, while $\mathrm{p}-\mathrm{mTOR} / \mathrm{mTOR}$ decreased $(P<0.01)$. Compared with group 2, the expression levels of LC-3II/ LC-3I and beclin 1 in the treatment groups decreased, while $\mathrm{p}-\mathrm{mTOR} /$ mTOR increased $(P<0.05$ or $P<0.01)$.

\section{Discussion}

This study is based on the theory of Chinese medicine, according to the pathogeny doctrine of TCM and western medicine, to establish the animal model of the syndrome of accumulation of cold fluid in the lung in chronic bronchitis asthma ${ }^{15}$. The syndrome of accumulation of cold fluid in the lung in chronic bronchitis asthma reflects two aspects of the disease syndrome: yang deficiency and spleen and lung qi deficiency. Qingxiang Z, Shaohong $Y^{16,17}$ induced asthma model by sensitizing and challenging with OVA, and established the model of syndrome of accumulation of cold fluid in the lung in chronic bronchitis asthma by drink, body cold and fatigue stimulation. The asthma model rats in our research show a few syndromes, such as rapid breathing, wheezing, mild cyanosis, loud wheezing can be heard in some rats, and there are white sticky secretions in the mouth and nose. We found model rats suffered body cold stimulation, appearing symptoms and signs of yang deficiency, for example, limbs not warm, nose, ears and tail pale, model rats suffered drink cold and fatigue stimulation appear symptoms and signs of spleen and lung qi deficiency, such as body emaciation, rough and lusterless fur or thinning, enuresis, loose stools, ecphysesis, mental fatigue, slow in reacting or agitated state, and weight loss, Symptoms and signs of model rats appear identically with the syndrome of accumulation of cold fluid in the lung in chronic bronchitis asthma in clinic.

The airway of asthmatic patients may be overreactive due to airway inflammation, showing sensitive and over strong contraction of the bronchial smooth muscle, resulting in airway constriction and increased airway resistance. It causes cough, chest tightness, wheezing, and other symptoms. The important features of bronchial asthma are airway obstruction and airway hyperresponsiveness. In experimenting, we determined that inspiratory resistance and expiratory resistance increased significantly, while lung ventilation compliance decreased significantly. It suggested modeling factors increase of pulmonary airway resistance in rats, inducing hyperresponsiveness of the airway. In the study of pathomorphological of lung tissue of model rats, there were a lot of inflammatory cell infiltration around the bronchus and epithelial cells of bronchial mucous membrane, especially lymphocytes and EOS, cilia adhered; there were inflammatory cells and increased mucus content in bronchial lumen; blood cells seeped, the alveolar walls are thickened; Pulmonary alveolus seeped much liquids, evenly ruptured and merged. The phenomenon was consistent with the pathomorphological features of clinical bronchial asthma.

According to the characteristic of bronchial asthma with the syndrome of accumulation of cold fluid in the lung, we drafted WYHY. It was composed of the classical prescription Xiao Qinglong Decoction with astragalus and ginseng. It has reached the therapeutic effect of warming yang for resolving fluid retention and freeing lung and relieving asthma. Ephedrine in ephedra in decoction has an obvious relaxant effect on bronchial smooth muscle, especially during bronchial spasm ${ }^{18}$. The volatile oil of Cassia twig has antiinflammatory effect on respiratory inflammation ${ }^{19}$. Licorice flavonoids, licorice extract, and glycyrrhetinic acid have remarkable preventing cough, eliminating phlegm effects. meanwhile, they strengthen anti-inflammatory effects by inhibiting macrophages producing PEG2 and IL-6 ${ }^{20,21}$. Rhizoma Zingiber asarum and Paeonia lactiflora show anti-inflammatory effects by inhibiting the production of proinflammatory mediators in macrophages and monocytes. While Asarum volatile oil may indeed relieve bronchial spasm. Alkaloids from Pinellia ternata can prevent cough, Eliminate phlegm by inhibiting cough center ${ }^{22-24}$. The effective components saponins and polysaccharides of ginseng and Astragalus have anti-inflammatory, antibacterial, and regulated smooth muscle activity effects. Meanwhile, they may protect macrophages to enhance its phagocytosis ${ }^{25}$. The pharmacological actions of the effective ingredients in the prescription indicate that WYHY can effectively inhibit the inflammatory reaction of the respiratory tract and reverse the pathological process of airway remodeling. 
TCM could treat bronchial asthma by blocking inflammation and reversing airway remodeling. In this study, we observed that WYHY can relieve the main symptoms and signs of model rats, such as shortness of breath, wheezing, secretion of airway mucus, improve lung function, inhibit the infiltration of inflammatory cells around the epithelial cells and alveoli (mainly EOS and lymphocyte), and reduce the e EOS induced cytokine, and correct the Th1/Th2 immune imbalance.

AHR is also a major symptom of asthma. After the treatment of WYHY, we determined that inspiratory resistance and expiratory resistance decreased significantly, while lung ventilation compliance increased significantly. Airway inflammation induction was one of the main causes of hyperresponsiveness ${ }^{26,27}$. EOS, lymphocytes, and macrophages are important inflammatory cells in asthma. Cytokines and chemokines induced by inflammatory cells in the airway aggravate airway hyperresponsiveness. After the treatment, the epithelial cells of the bronchial mucosa of the rat showed slight swelling and hyperplasia. A small amount of mucus and inflammatory cells were found in the bronchial tube. The alveolar wall was slightly thickened, and a small amount of EOS and lymphocyte infiltration could be seen. They pointed out that WYHY can reduce the number of EOS and lymphocyte inflammatory response and relieve airway hyperresponsiveness.

TGF- $\beta_{1}$ is a major regulator of airway remodeling. During asthma attacks, macrophages, epithelial cells, smooth muscle cells, and EOS in the airway secrete a large amount of TGF- $\beta_{1}$. TGF- $\beta 1$ stimulates proliferation and hypertrophy of airway smooth muscle cells, induces the division and proliferation of fibroblasts, and promotes to transform fibroblasts into myofibroblasts ${ }^{28}$. The expression of TGF- $\beta_{1}$ was positively correlated with the thickness of airway basement membrane, the number of fibroblasts, and (or) the severity of asthma attacks ${ }^{29}$. In this study, we observed that there were high levels of TGF- $\beta 1$ in blood and asthma attacks heavily. After treatment of WYHY, the level of TGF- $\beta 1$ was reduced, the clinical symptoms were alleviated, it is possible that the decoction could reverse the level of TGF- $\beta 1$.

A large number of studies have confirmed that Th2 cells play an important role in the pathogenesis of asthma. IL-13 is one of Th2 cytokines, which may cause inflammatory cells to adhere to the vascular endothelium, and then transmembrane wandering gather around the airway ${ }^{30}$. Meanwhile, it can inhibit the apoptosis of EOS and B cells, so that airway inflammation persists ${ }^{31}$. IL-13 induces and activates fibrotic medium TGF- $\beta$, which stimulates myofibroblast proliferation and participates in airway fibrosis ${ }^{32}$. The

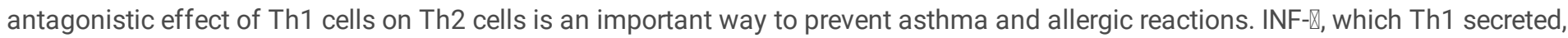
has the function of phagocytosis of microorganisms in the cell that can downregulate the expression of EOS chemokine receptor and inhibit EOS infiltration ${ }^{33}$. IFN- $\llbracket$ can also inhibit the proliferation of fibroblasts and the synthesis of collagen, promote the apoptosis of fibroblasts, and repair the damaged alveolar epithelium, and reduce the expression of TGF- $\beta^{34}$. Therefore, IFN- $\rrbracket$ plays an important role in suppressing airway inflammatory cell infiltration and reversing airway remodeling. TNF- $\mathbb{Q}$ is a proinflammatory cytokine, produced by mononuclear macrophage ${ }^{35}$, which makes the adhesion molecules of endothelial cells highly expressed. These adhesion molecules combine with the corresponding receptors on the inflammatory cells, thereby promoting the accumulation of inflammatory cells across

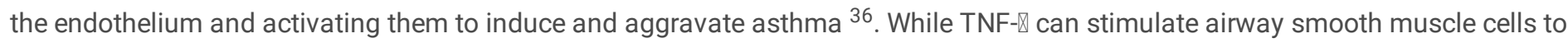
secrete endothelin, which exacerbate the contraction of smooth muscle and cause airway remodeling.

Our experimental results showed that the expression of IL-13 and TNF- $₫$ in the peripheral blood of model rats decreased significantly,

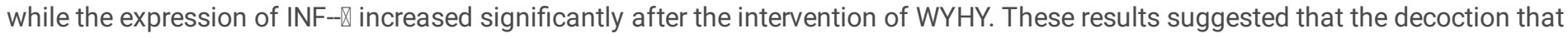

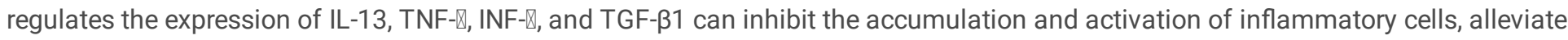
airway inflammation, slow down the contraction of smooth muscle, and reverse the airway remodeling.

\section{Conclusion}

WYHY decreased the expression of IL-13 and TNF- $\bigotimes$, TGF- $\beta 1$ in peripheral blood, while increased the expression of INF- $\mathrm{Z}$. WYHY can relieve the main symptoms and signs of model rats, such as shortness of breath, wheezing, secretion of airway mucus, improve lung function, inhibit the infiltration of inflammatory cells, slow down the contraction of smooth muscle, and reverse airway remodeling.

\section{Declarations}

\section{Ethics Approval}


The study was approved by the comments of the laboratory animal care committee of Shandong University of Traditional Chinese Medicine (NO. SDUTCM2015020101).

\section{Consent to publish}

The authors declare that they all agree to publish the paper.

\section{Availability of data and materials}

The datasets used or analysed during the current study are available from the corresponding author on reasonable request.

\section{Conflict of Interest}

The authors declare that they have no known competing financial interests or personal relationships that could have appeared to influence the work reported in this paper.

\section{Fundings}

This study was supported by the National Natural Science Foundation of China (NSFC, Nos. 81470189, 81774169, and 81874419).

\section{Authors' Contributions}

Zhang Qingxiang designed the experiment scheme, guided the whole experiment, and reviewed and revised the manuscript. Yan Peizheng and Zhu Qingjun conducted the experiments and wrote the draft of manuscript. Chang Xing, Meng Qingyan and Liu Yan conducted the experiments and data analysis, and approved the final version of the manuscript.

\section{Acknowledgements}

Not applicable

\section{References}

1. Croisant S. Epidemiology of asthma: prevalence and burden of disease. Advances in experimental medicine and biology. 2014;795:17-29.

2. Bousquet J, Schünemann HJ, Samolinski B, et al. Allergic Rhinitis and its Impact on Asthma (ARIA): achievements in 10 years and future needs. The Journal of allergy and clinical immunology. Nov 2012;130(5):1049-1062.

3. Venero SJ, González FJ, Suárez R, Fabré D, Fernández HM. Epidemiology of asthma mortality in cuba and its relation to climate, 1989 to 2003. MEDICC review. Jul 2008;10(3):24-29.

4. Zhi Y, Huang H, Liang L. MFG-E8/integrin $\beta 3$ signaling contributes to airway inflammation response and airway remodeling in an ovalbumin-induced murine model of asthma. Journal of cellular biochemistry. Nov 2018;119(11):8887-8896.

5. Choi Y, Le Pham D, Lee DH, Lee SH, Kim SH, Park HS. Biological function of eosinophil extracellular traps in patients with severe eosinophilic asthma. Experimental \& molecular medicine. Aug 16 2018;50(8):104.

6. Martin JG. Airway smooth muscle may drive mucus hypersecretion in asthma. The European respiratory journal. Aug 2018;52(2)

7. Kong L, Zhang H, Cao Y, et al. The Anti-Inflammatory Effects of Invigorating Kidney and Supplementing Qi Chinese Herbal Formulae in Asthma Patients. Evidence-based complementary and alternative medicine : eCAM. 2017;2017:3754145.

8. Xu L, Li J, Zhang Y, Zhao P, Zhang X. Regulatory effect of baicalin on the imbalance of Th17/Treg responses in mice with allergic asthma. Journal of ethnopharmacology. Aug 17 2017;208:199-206.

9. Wang Z, Li J, Xie Y, Yu X, Zhang Z. Traditional Chinese medicine ZHENG identification of bronchial asthma: Clinical investigation of 2500 adult cases. Complementary therapies in medicine. Feb 2017;30:93-101. 
10. Zhang $Y$, Jing $Y$, Qiao J, et al. Activation of the mTOR signaling pathway is required for asthma onset. Scientific reports. Jul 3 2017;7(1):4532.

11. Yang YG, Tian WM, Zhang H, Li M, Shang YX. Nerve growth factor exacerbates allergic lung inflammation and airway remodeling in a rat model of chronic asthma. Experimental and therapeutic medicine. Nov 2013;6(5):1251-1258.

12. Kabiri Rad M, Neamati A, Boskabady MH, Mahdavi-Shahri N, Mahmoudabady M. The preventive effect of Brassica napus L. oil on pathophysiological changes of respiratory system in experimental asthmatic rat. Avicenna journal of phytomedicine. Winter 2013;3(1):56-63.

13. J.F.Sun. Animal Experiment Methodology. Beijing, China: Peoples Medical Publishing House; 2001.

14. Chen $\mathrm{M}, \mathrm{Gu} \mathrm{H}, \mathrm{Ye} \mathrm{Y}$, et al. Protective effects of hesperidin against oxidative stress of tert-butyl hydroperoxide in human hepatocytes. Food and chemical toxicology : an international journal published for the British Industrial Biological Research Association. Oct 2010;48(10):2980-2987.

15. Grainge C, Park JA. Inflammatory insights into airway remodelling in asthma. Respirology (Carlton, Vic.). Dec 2018;23(12):1084-1085.

16. Fredriksson K, Fielhaber JA, Lam JK, et al. Paradoxical effects of rapamycin on experimental house dust mite-induced asthma. PloS one. 2012;7(5):e33984.

17. Zhu C, Xia L, Li F, et al. mTOR complexes differentially orchestrates eosinophil development in allergy. May 2 2018;8(1):6883.

18. Han J, Li S, Zhao Y, et al. [Acupuncture for TGF- $\beta 1$ expression in lung tissues of asthma rats]. Zhongguo zhen jiu $=$ Chinese acupuncture \& moxibustion. Jul 12 2017;37(7):741-746.

19. Ding F, Liu B, Zou W, et al. LPS Exposure in Early Life Protects Against Mucus Hypersecretion in Ovalbumin-Induced Asthma by Down-Regulation of the IL-13 and JAK-STAT6 Pathways. Cellular physiology and biochemistry : international journal of experimental cellular physiology, biochemistry, and pharmacology. 2018;46(3):1263-1274.

20. Keyhanmanesh R, Rahbarghazi R, Ahmadi M. Systemic Transplantation of Mesenchymal Stem Cells Modulates Endothelial Cell Adhesion Molecules Induced by Ovalbumin in Rat Model of Asthma. Inflammation. Dec 2018;41(6):2236-2245.

21. Zhang Y, Xu B, Luan B, et al. Myeloid-derived suppressor cells (MDSCs) and mechanistic target of rapamycin (mTOR) signaling pathway interact through inducible nitric oxide synthase (iNOS) and nitric oxide (NO) in asthma. American journal of translational research. 2019;11(9):6170-6184.

22. S. Y, C. C. pharmacology of Chinese materia medica. Shanghai, China: Shanghai Science and Technology Press; 2012.

23. Wang M, Zhang M, Tang Q, Li X. Influence of honey-roasting on the main pharmacological activities and the water-soluble active glycosides of licorice. African journal of traditional, complementary, and alternative medicines : AJTCAM. 2012;9(2):189-196.

24. Kim JY, Park SJ, Yun KJ, Cho YW, Park HJ, Lee KT. Isoliquiritigenin isolated from the roots of Glycyrrhiza uralensis inhibits LPS-induced iNOS and COX-2 expression via the attenuation of NF-kappaB in RAW 264.7 macrophages. European journal of pharmacology. Apr 14 2008;584(1):175-184.

25. Xu Y, Cao C, Shang M, et al. [Assessment on anti-nociception and anti-inflammation pharmacodynamics of Asarum heterotropoides var. mandshuricum and Asarum sieboldii]. Zhongguo Zhong yao za zhi = Zhongguo zhongyao zazhi = China journal of Chinese materia medica. Mar 2012;37(5):625-631.

26. Y. J, M. C, J. T. Advances in studies on chemical constituents and pharmacological effects of Paeonia lactiflora Pall. Chinese Journal of Pharmacology and Toxicology. 2013;27(4):745-750.

27. q. X, W. Y, D. Z, X. Y. Research Progress on influential factors and pharmacological actions of total alkaloids in Pinellia ternate. China Pharmaceuticals. 2016;25(3):123-126. 
28. Coutts A, Chen G, Stephens N, et al. Release of biologically active TGF-beta from airway smooth muscle cells induces autocrine synthesis of collagen. American journal of physiology. Lung cellular and molecular physiology. May 2001;280(5):L999-1008.

29. Vignola AM, Chanez P, Chiappara G, et al. Transforming growth factor-beta expression in mucosal biopsies in asthma and chronic bronchitis. American journal of respiratory and critical care medicine. Aug 1997;156(2 Pt 1):591-599.

30. Elias JA, Zheng T, Lee CG, et al. Transgenic modeling of interleukin-13 in the lung. Chest. Mar 2003;123(3 Suppl):339s-345s.

31. Hara K, Kondo M, Tsuji M, Takeyama K, Tamaoki J. Clarithromycin suppresses IL-13-induced goblet cell metaplasia via the TMEM16A-dependent pathway in guinea pig airway epithelial cells. Respiratory investigation. Jan 2019;57(1):79-88.

32. Yao L, Wang S, Wei P, et al. Huangqi-Fangfeng protects against allergic airway remodeling through inhibiting epithelialmesenchymal transition process in mice via regulating epithelial derived TGF- $\beta 1$. Phytomedicine : international journal of phytotherapy and phytopharmacology. Nov 2019;64:153076.

33. Kim CK, Choi J, Callaway Z, lijima K, Volcheck G, Kita H. Increases in airway eosinophilia and a th1 cytokine during the chronic asymptomatic phase of asthma. Respiratory medicine. Oct 2010;104(10):1436-1443.

34. Kassa B, Mickael C, Kumar R, et al. Paclitaxel blocks Th2-mediated TGF- $\beta$ activation in Schistosoma mansoni-induced pulmonary hypertension. Pulmonary circulation. Jan-Mar 2019;9(1):2045894018820813.

35. Dash S, Sarashetti PM, Rajashekar B, Chowdhury R, Mukherjee S. TGF- $\beta 2$-induced EMT is dampened by inhibition of autophagy and TNF-a treatment. Oncotarget. Jan 19 2018;9(5):6433-6449.

36. Maes T, Cobos FA, Schleich F, et al. Asthma inflammatory phenotypes show differential microRNA expression in sputum. The Journal of allergy and clinical immunology. May 2016;137(5):1433-1446.

\section{Figures}


A

Drink cld, Body cold, Fatigue stimulation

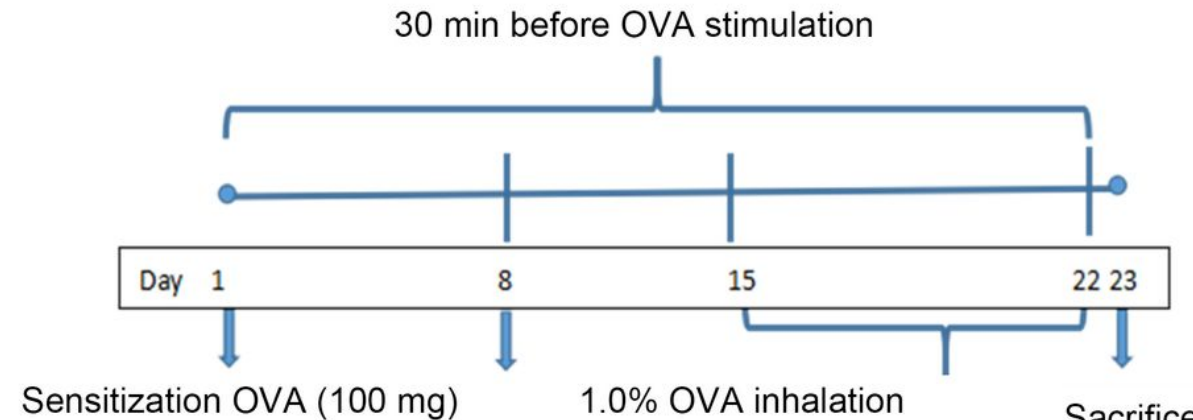

$+\mathrm{Al}(\mathrm{OH}) 3(100 \mathrm{mg}) \quad$ (30 $\mathrm{min}$ per day, $3 \mathrm{ml} / \mathrm{min})$

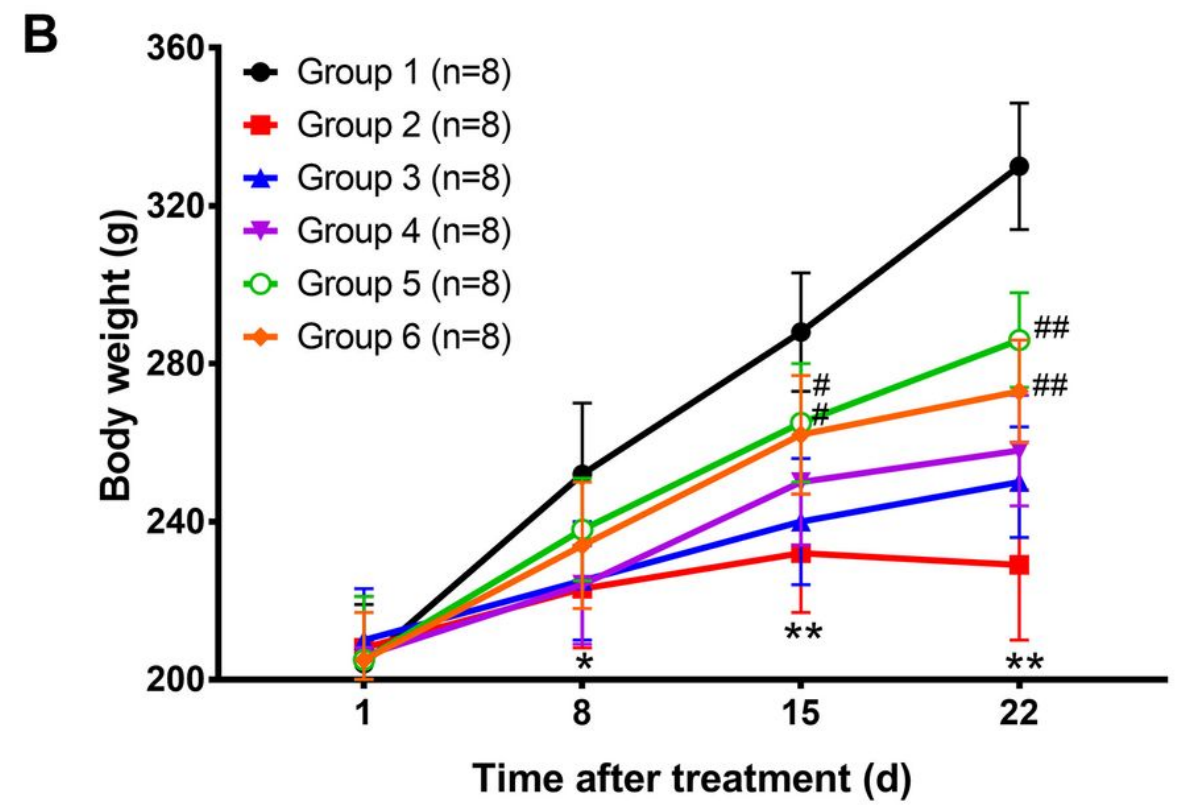

Figure 1

The procedure to establish an asthma model and the body weight of rats in different stages. A: The procedure to establish an asthma model. B: The body weight of rats. Those in group 1 increased steadily, while Weight growth of the other five groups were relatively slower. At $15^{\text {th }}$ and $22^{\text {nd }}$ day, Weight of rats in group 4,5 and 6 were higher than group 2 . Compared with group $1,{ }^{\star} P<0.05,{ }^{\star \star} P<0.01$; Compared with group $2,{ }^{\#} P<0.05,{ }^{\# \#} P<0.01$. 


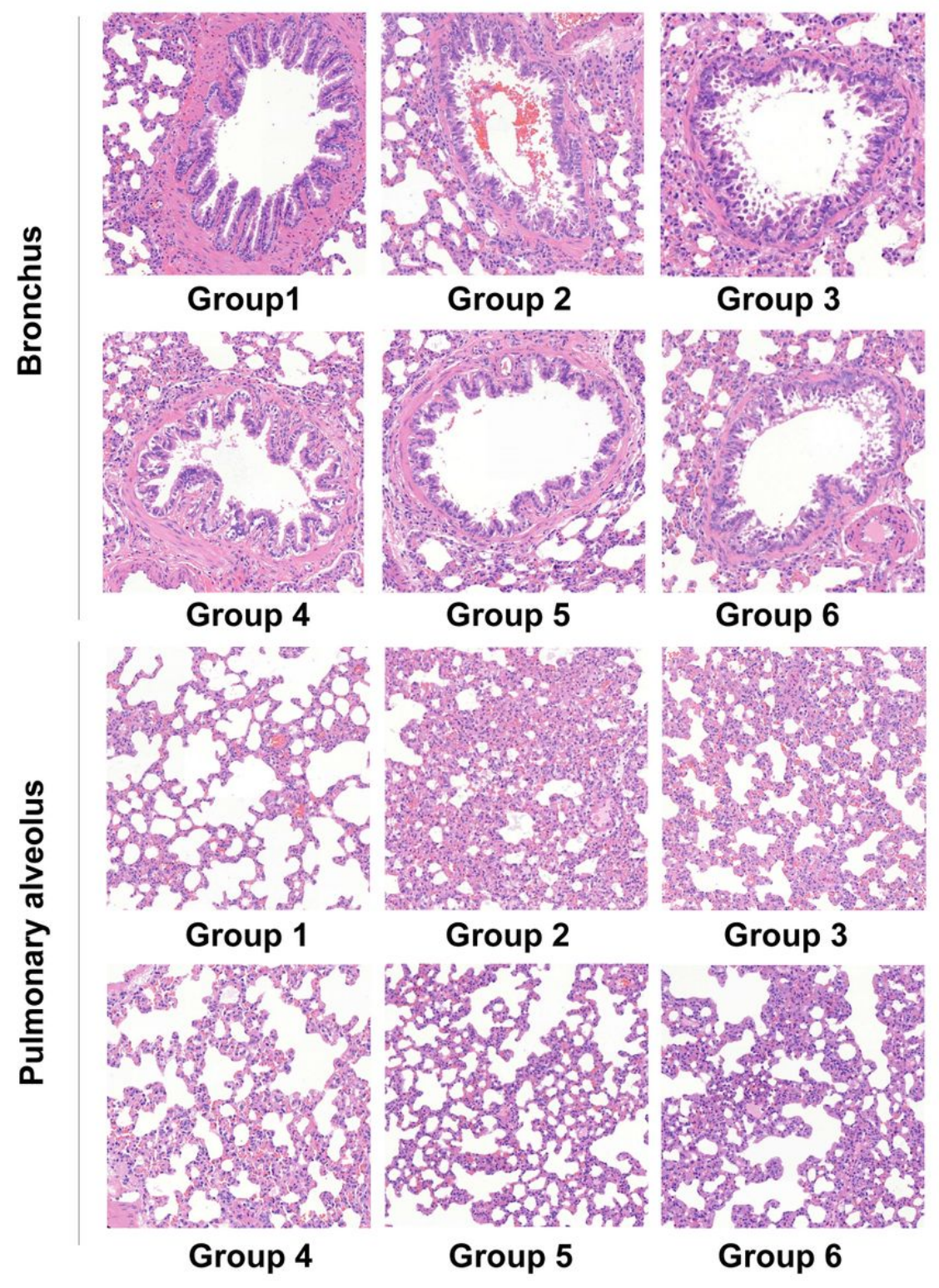

Figure 2

The pathological features of bronchus and pulmonary alveolus observed after HE staining (200x). Group 1: normal group, Group 2: model control group, Group 3: rapamycin group, Group 4: Xiaoqinglong Decoction group, Group 5: WYHY group, Group 6: astragalus granules group. 
A
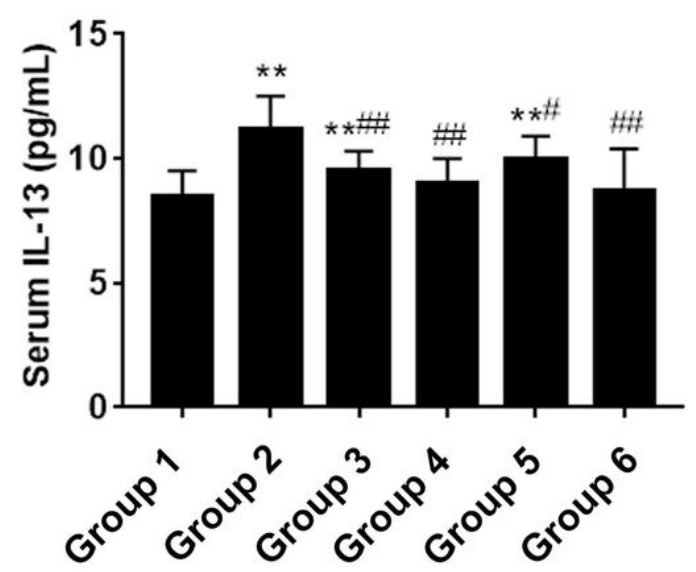

C

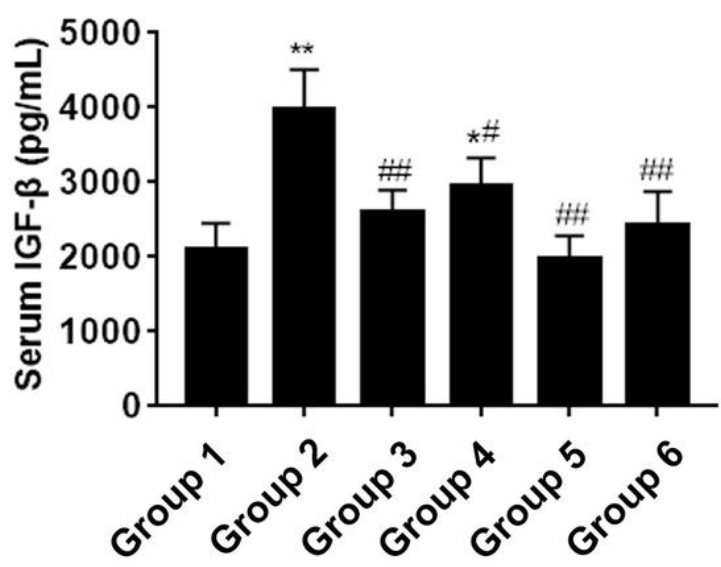

B

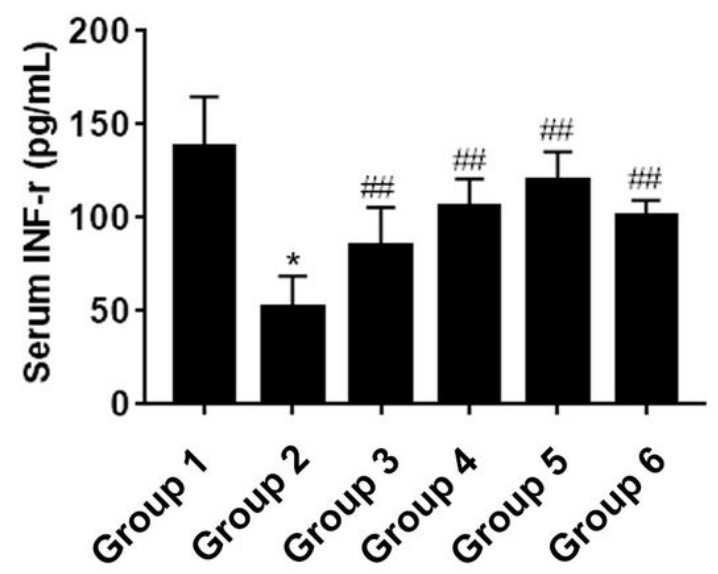

D

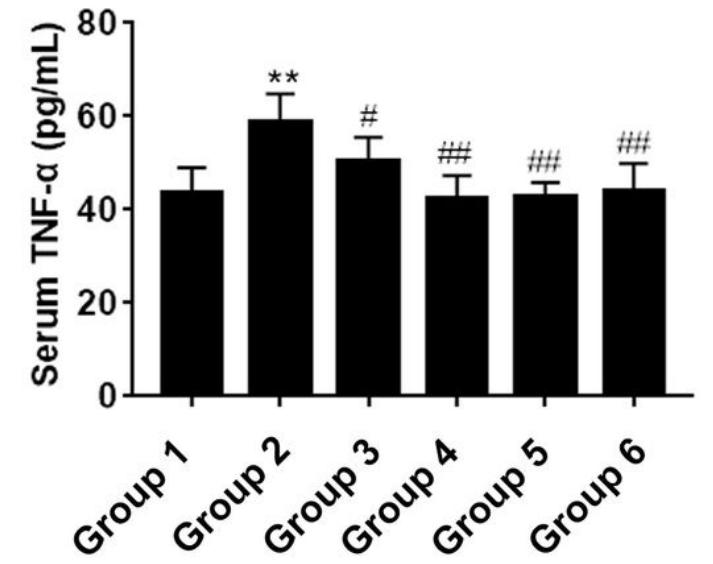

Figure 3

Serum levels of IL-13, INF- $\gamma$, TGF- $\beta 1$, and TNF- $a$ in chronic bronchitis asthma rats. A: IL-13, B: INF- $\gamma$, C: TGF- $\beta$, D: TNF-a. Group 1: normal group, Group 2: model control group, Group 3: rapamycin group, Group 4: Xiaoqinglong Decoction group, Group 5: WYHY group, Group 6: astragalus granules group.

Compared with group $1,{ }^{\star} P \otimes 0.05,{ }^{\star \star} P \otimes 0.01 \llbracket$ Compared with group $2,{ }^{\#} P \otimes 0.05,{ }^{\# \#} P \otimes 0.01$. 


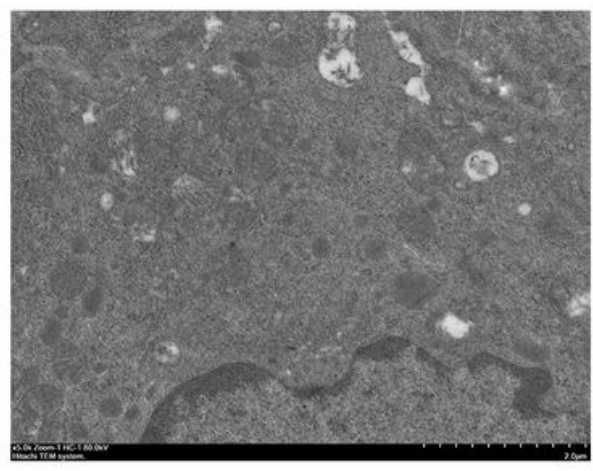

Group 1

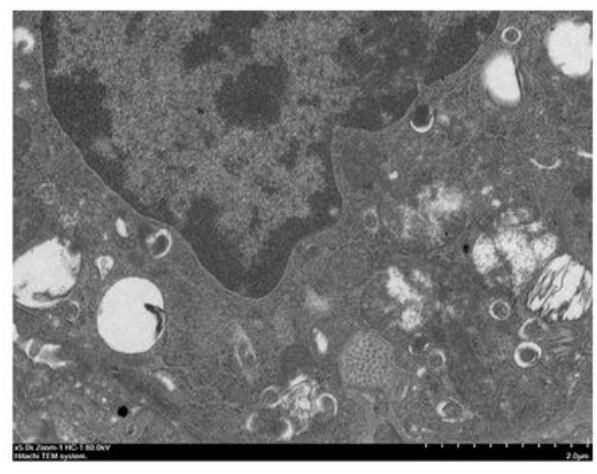

Group 4

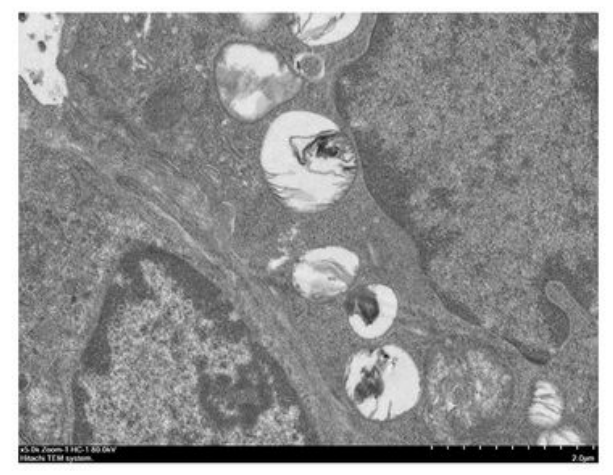

Group 2

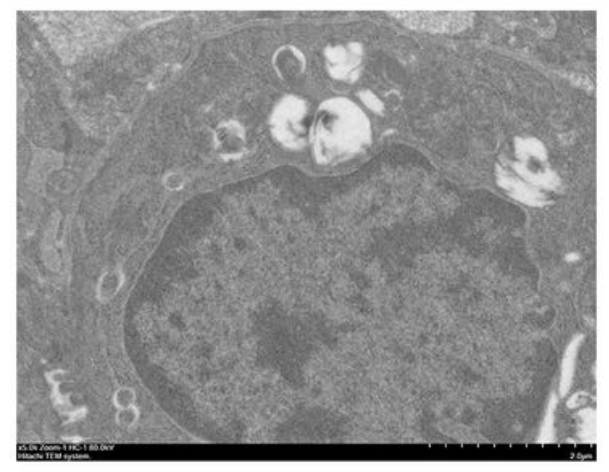

Group 5

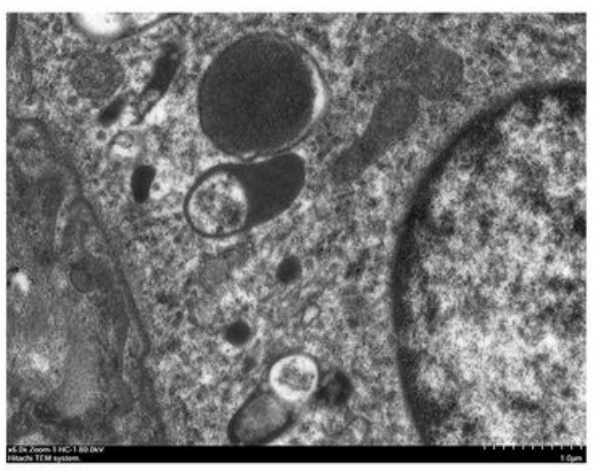

\section{Group 3}

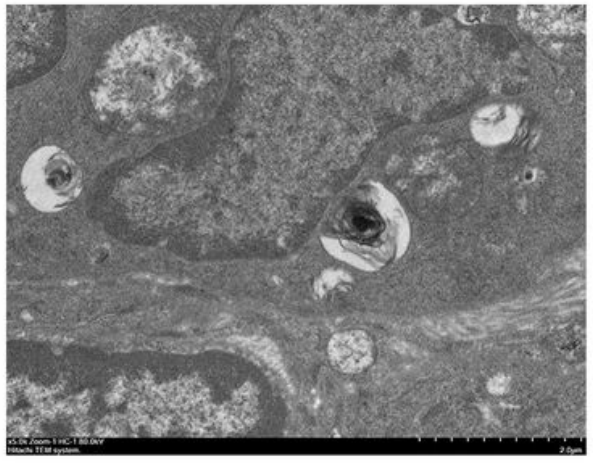

Group 6

Figure 4

The autophagosomes in the lung tissues were observed in TEM (200x). Group 1: normal group, Group 2: model control group, Group 3: rapamycin group, Group 4: Xiaoqinglong Decoction group, Group 5: WYHY group, Group 6: astragalus granules group. 


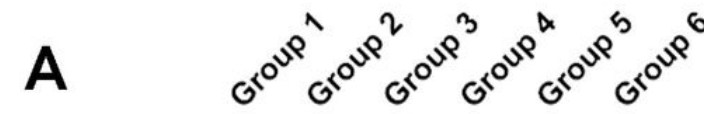

Anti-LC3ㅍ/ I

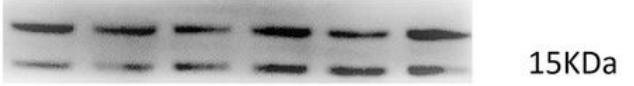

GAPDH

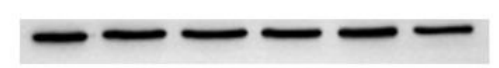

36KDa

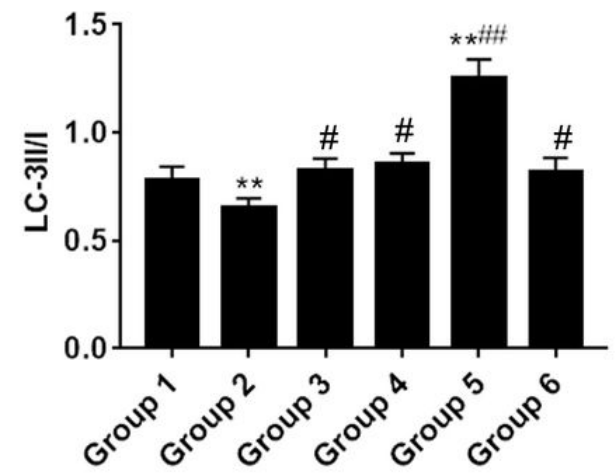

B

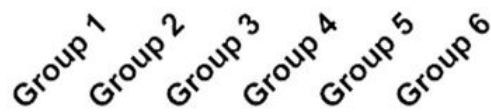

Beclin 1

$52 \mathrm{KDa}$

GAPDH

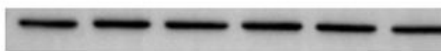

36KDa

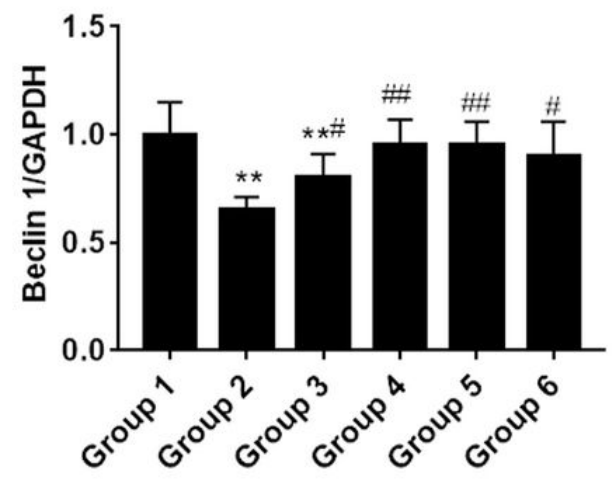

C

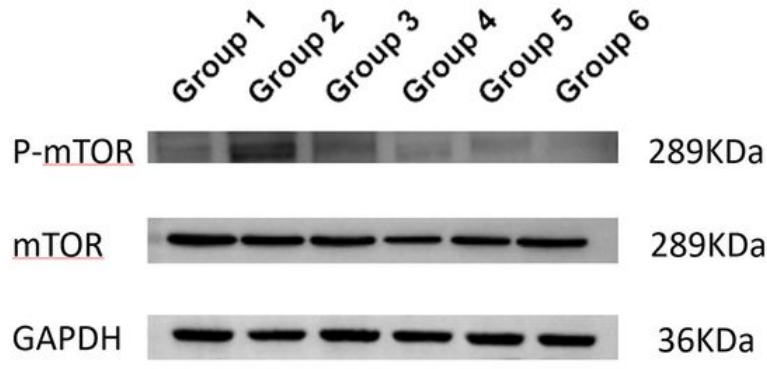

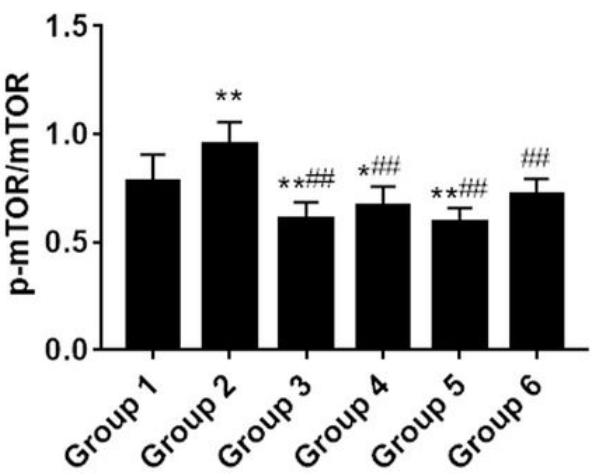

Figure 5

The protein expression of autophagy associated proteins were determined by WB. A囚LC-3II/3I, B: Beclin, C: m-TOR/TOR. Group 1: normal group, Group 2: model control group, Group 3: rapamycin group, Group 4: Xiaoqinglong Decoction group, Group 5: WYHY group, Group 6: astragalus granules group. Compared with group $1,{ }^{\star \star} P \otimes 0.01 \otimes C o m p a r e d$ with group $2,{ }^{\#} P \otimes 0.05,{ }^{\# \#} P \otimes 0.01$.

\section{Supplementary Files}

This is a list of supplementary files associated with this preprint. Click to download.

- supplementarymaterialforreference.pdf 